\title{
Hierarchical shared transfer learning for biomedical named entity recognition
}

\author{
Zhaoying Chai ${ }^{1}$, Han Jin ${ }^{1}$, Shenghui Shi ${ }^{{ }^{*}}$, Siyan Zhan ${ }^{2^{*}}$, Lin Zhuo ${ }^{3}$ and Yu Yang ${ }^{4}$
}

\author{
*Correspondence: \\ shish@mail.buct.edu.cn; \\ siyan-zhan@bjmu.edu.cn \\ ${ }^{1}$ College of Information \\ Science and Technology, \\ Beijing University of Chemical \\ Technology, Beijing, China \\ ${ }^{2}$ School of Public Health, \\ Peking University, Beijing, \\ China \\ Full list of author information \\ is available at the end of the \\ article
}

\begin{abstract}
Background: Biomedical named entity recognition (BioNER) is a basic and important medical information extraction task to extract medical entities with special meaning from medical texts. In recent years, deep learning has become the main research direction of BioNER due to its excellent data-driven context coding ability. However, in BioNER task, deep learning has the problem of poor generalization and instability.

Results: we propose the hierarchical shared transfer learning, which combines multitask learning and fine-tuning, and realizes the multi-level information fusion between the underlying entity features and the upper data features. We select 14 datasets containing 4 types of entities for training and evaluate the model. The experimental results showed that the F1-scores of the five gold standard datasets BC5CDR-chemical, BC5CDR-disease, BC2GM, BC4CHEMD, NCBI-disease and LINNAEUS were increased by $0.57,0.90,0.42,0.77,0.98$ and -2.16 compared to the single-task XLNet-CRF model. BC5CDR-chemical, BC5CDR-disease and BC4CHEMD achieved state-of-the-art results. The reasons why LINNAEUS's multi-task results are lower than single-task results are discussed at the dataset level.
\end{abstract}

Conclusion: Compared with using multi-task learning and fine-tuning alone, the model has more accurate recognition ability of medical entities, and has higher generalization and stability.

Keywords: BioNLP, Biomedical named entity recognition, Transfer learning, Permutation language model, Conditional random field

\section{Background}

Biomedical information extraction is an important tool to handle the unmarked medical literature of exponential growth, and the extracted information has important value for medical research [1]. Biomedical named entity recognition (BioNER) is a basic task in biomedical information extraction to extract interested entities such as diseases, drugs, genes/proteins from complex, unstructured medical texts [2].

With the efforts of many researchers, more and more deep learning networks have emerged, ranging from Convolutional Neural Network (CNN) [3], Long Short-Term Memory Networks (LSTM) [4], to Transformers-based BERT language models in BioNER. But single-task learning has always had the problem of poor generalization in

(c) The Author(s), 2021. Open Access This article is licensed under a Creative Commons Attribution 4.0 International License, which permits use, sharing, adaptation, distribution and reproduction in any medium or format, as long as you give appropriate credit to the original author(s) and the source, provide a link to the Creative Commons licence, and indicate if changes were made. The images or other third party material in this article are included in the article's Creative Commons licence, unless indicated otherwise in a credit line to the material. If material is not included in the article's Creative Commons licence and your intended use is not permitted by statutory regulation or exceeds the permitted use, you will need to obtain permission directly from the copyright holder. To view a copy of this licence, visit http:// creativecommons.org/licenses/by/4.0/. The Creative Commons Public Domain Dedication waiver (http://creativecommons.org/publi cdomain/zero/1.0/) applies to the data made available in this article, unless otherwise stated in a credit line to the data. 
BioNER task. Mehmood and others [5] proposed multi-task learning based on CNN and LSTM to improve the generalization of the model, but the results was difficult to go beyond single-task learning based on Transformers model and unstable. In order to improve the generalization of the model, we do multi-task learning based on Transformers, but the experimental results once again verify that simple multi-task learning results are not stable, some datasets are improved, but some datasets are not better than single-task learning. Therefore, we propose the hierarchical shared transfer learning, which combines multi-task learning with single-task learning, which not only allows the model to have high accuracy, but also improves the generalization and stability of the model.

We used XLNet [6] based on Self-Attention Permutation Language Model (PLM) to replace BERT as encoder in the pre-training phase, avoiding the problem of input noise from autoencoding language model (AutoEncoder LM). When fine-tuning the BioNER task, we decode the output of the XLNet model with conditional random field (CRF) decoder. Because XLNet uses tagged input, the connection layer between XLNet and CRF is tuned with Label [X]. For multi-tasking training, we split the datasets and combined similar entity datasets. We share all the parameters of the XLNet-CRF during training, and then evaluate the effects of each dataset separately. We refer to the model that shares all XLNet-CRF model parameters for multi-tasking learning as MTLXC. However, the experimental results show that the learning of MTL-XC is unstable. In order to solve this problem, we propose hierarchical shared transfer learning. We divide the parameters of XLNet-CRF model into shared parts and task-specific parts. The shared portion is for multi-task learning, the specific task portion is for single-task learning, and we refer to this new model as MTL-LS, with good results.

- Permutation language model and conditional random field were combined.

- Aiming at the instability of multi-task learning in BioNER, a hierarchical shared transfer learning method combining multi-task learning and single-task learning was proposed.

- Through the analysis of the physical relationship between the training set, the test set and the training effect, the source of the data-level error was obtained.

The source code is detailed in : https://github.com/pwldj/MTL-BioNER.

\section{Related work}

\section{Transfer learning}

Transfer learning has gained general attention in the field of machine learning in recent years [7] by transferring knowledge from relevant tasks that have been learned to improve new tasks [8]. Transfer learning can be divided into instance-based transfer, feature representation transfer, parameter transfer and relational knowledge transfer [9]. Where parameter transfer is already commonly used in NLP tasks, it is assumed to share some parameters between source tasks and target tasks, or to share a prior distribution of model hyperparameters [10]. This also enables good accuracy when transferring the original model to the new domain $[11,12]$. However, there are also problems with negative transfer. For the problem of negative transfer, Wang et al. quantifies the similarity between target domain and source domain by calculating the 
affinity matrix of gene, and automatically learns the fusion network of target cancer [13]. Tao et al. proposes that the REFERENCE algorithm makes use of the semantic correlation between source sample and target task, rather than the task/sample similarity [14].

\section{Multi-tasking}

Transfer learning, which can be attributed to making the most of all available information, has become an important research direction in Biomedical named entity recognition [15, 16]. Multi-tasking learning (MTL) [17] is a major form of transfer learning that involves learning part of a model or the whole on multiple similar tasks, thereby enhancing the recognition of the model on a particular type of task. Crichton et al., first applied it to the field of BioNER, and by using convolutional neural networks and different shared layer methods, it achieved more than single-task learning (STL) on some entity types [18]. However, the performance on the remaining entity types was not satisfactory. Then, the LSTM gradually became the mainstream of BioNER [19]. Wang et al. [20] achieved an improvement over single-task learning by sharing different parameters embedded in words and character levels. Mehmood et al. use stackLSTM to share underlying LSTM to multiple similar tasks, while upper-level LSTM trains for different tasks [5]. However, Zuo and Zhang train as a shared layer except for CRF, which trains separately for each task [21].

\section{Fine-tune}

Fine-tuning is another way of transferring learning, pre-training a large amount of unseen data before applying the pre-training model to other specific downstream tasks. Fine-tuning greatly promotes the study of natural language processing [22, 23], and the multi-head self-attention mechanism solves the disadvantages of the unidirectional LSTM model [24]. The BERT language model based on multi-head attention mechanism proposed by Devlin has achieved the most advanced results in many tasks [25]. The BioBERT model and the PubMedBert model have achieved significant improvement in many biomedical tasks by pre-training BERT models using medical materials and fine-tuning them [26, 27]. Based on the BERT model, multi-task learning is used to train multiple medical text mining tasks. But it has also been found that multi-task is not always effective [28]. But the BERT model produces noise during the pre-training phase that reduces the recognition of each word element. The XLNet language model improves the pre-training process of the BERT model [6]. XLNet combines the autoencoder and autoregressive language models and proposes that the PLM can effectively suppress mask noise by predicting the different permutation of the same input sequence. XLNet outperformed the BERT model on 20 natural language processing tasks. The effectiveness of the Distributional Hypothesis of XLNet can acquire common sense and the structure of language from the statistical law of corpus. Its modeling approach from "unidirectional" context to "bidirectional" context and from "short-range" dependency to "long-range" dependency makes XLNet the most refined model for context modeling today. So our research revolves around XLNet. 


\section{Materials and methods}

\section{XLNet-CRF architecture}

XLNet is a permutation language model. In the pre-training phase, the noise in BERT model is eliminated by using the two-stream self-attention. At the fine-tuning phase, there is little difference between XLNet and the BERT model, both of which can be considered multi-headed self-attention language models.

We have decoded the output of the XLNet model using the CRF decoder. Figure 1 shows the XLNet-CRF architecture from a fine-tuned perspective. First, the text is serialized, and the input sequence is defined as $X=\left[x_{1} \ldots x_{t}\right]$, where $t$ is the length of the input sequence. The input sequence in XLNet is generated by the SentiencePiece [20] based on the input text. Then, after the $X$ has been word-embedded, each input character is mapped to a vector, forming the sequence $H^{0}=\left[h_{1}^{0} \ldots h_{t}^{0}\right]$ as input to the multi-header attention model. Finally, the output vector of the final XLNet model is $H^{n}=\left[h_{1}^{n} \ldots h_{t}^{n}\right]$ after the attention model is linked by the $n$ layer residue. The entity label for each character entered, corresponding to the input, is treated as $Y=\left[y_{0} \ldots y_{t}\right]$. Defines an entity label collection as $l \in 1,2 \ldots L, L$ is the total number of target identification tag sets, so BioNER tasks can be considered classification tasks that predict $Y$ based on $X$. Given the continuity of entity labels, the CRF is used as the decoding layer to select the most appropriate label from the label collection. $A$ is defined as a transition matrix to modify the current forecast based on previous label information. Therefore, the label forecast score is defined a

$$
S_{(X, y)}=\sum_{i=0}^{L} A_{y_{i}, y_{i}+1}+\sum_{i=1}^{L} P_{i, y_{i}}
$$

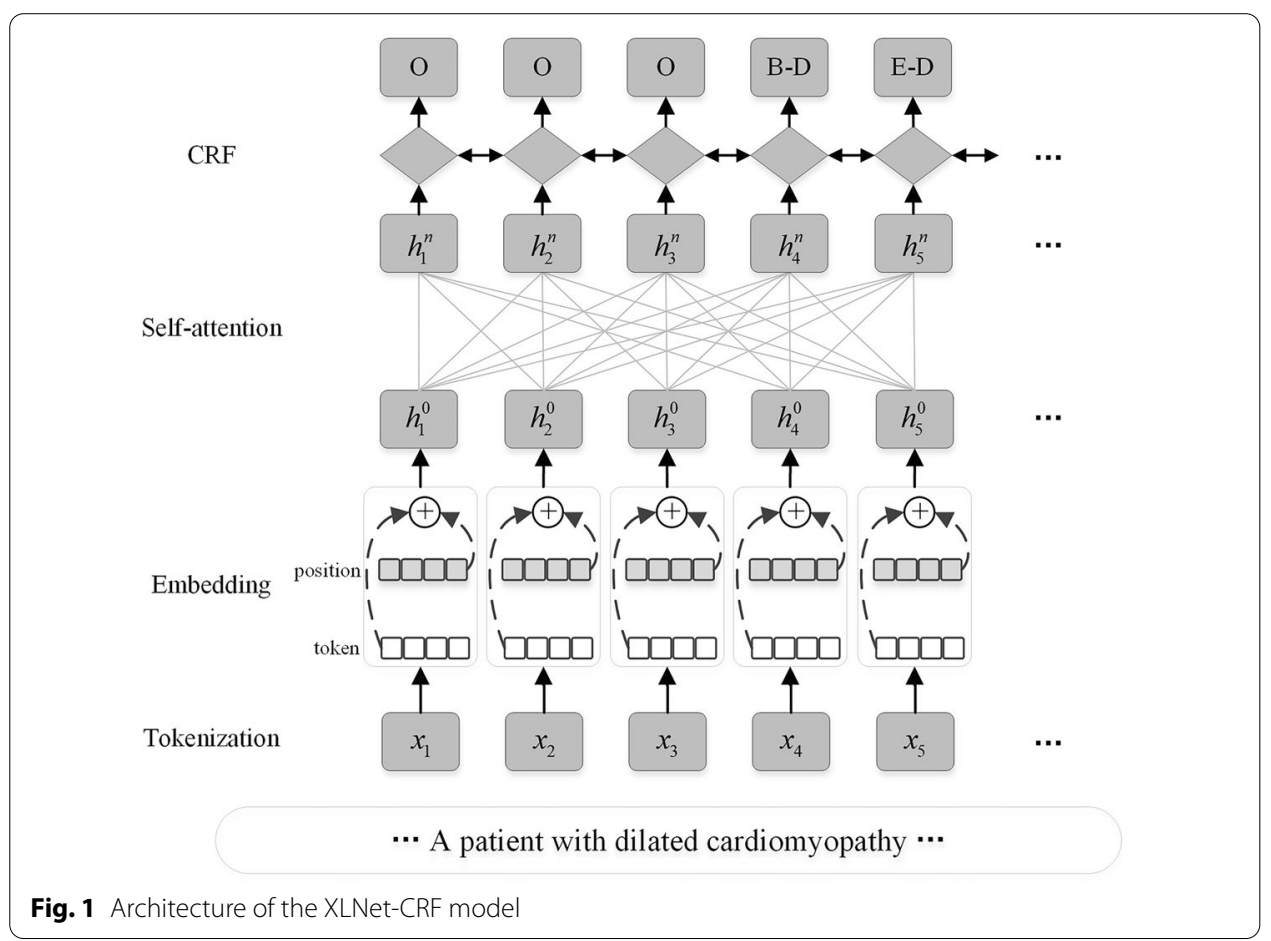


After softmax standardizes the label score, the conditional probability for each word element can be obtained. At the prediction and evaluation stage, the Viterbi algorithm [29] is used as the reasoning for the final prediction results.

\section{Multi-task learning}

The general deep learning model can fit the training target data highly. However, even for datasets of the same type of entity, it is still difficult to apply models trained for one dataset directly to another dataset [30]. On the one hand, when only one data set is targeted, there is inevitably an out-of-vocabulary (OOV). To some extent, the problem of OOV is mitigated by the paraphrasing of sentences. On the other hand, over-fitting is common for models that train on only one dataset. With the limited size of a single dataset, multi-tasking learning is an effective way to improve the generalization of models, while avoiding the over-fitting of models trained on a single dataset. Multi-tasking is the training of shared parameters on multiple tagged datasets where similar entities exist. In this paper, fine-tuning is integrated into multi-task learning. Pre-trained XLNet with common text data is used as an initialization parameter, while CRF model parameters are initialized randomly. On this basis, similar entity datasets are combined for training and the effects of each dataset are evaluated separately, corresponding to which single task learning is defined as fine-tuning training for each dataset on a pre-training model basis. We divided 14 datasets into four categories for multi-task learning. We share all the parameters of XLNet-CRF for multi-task, and we call this model MTL-XC.

\section{Hierarchical shared transfer learning}

We trained in single-task learning and multi-task learning respectively to evaluate the effectiveness of multi-task learning and found that the results were not as good as expected.

Inspired by the work of Mehmood et al., we proposed the MTL-LS (layer slicing) model. As shown in Fig. 2, we divide the XLNet-CRF model into shared and task-specific sections

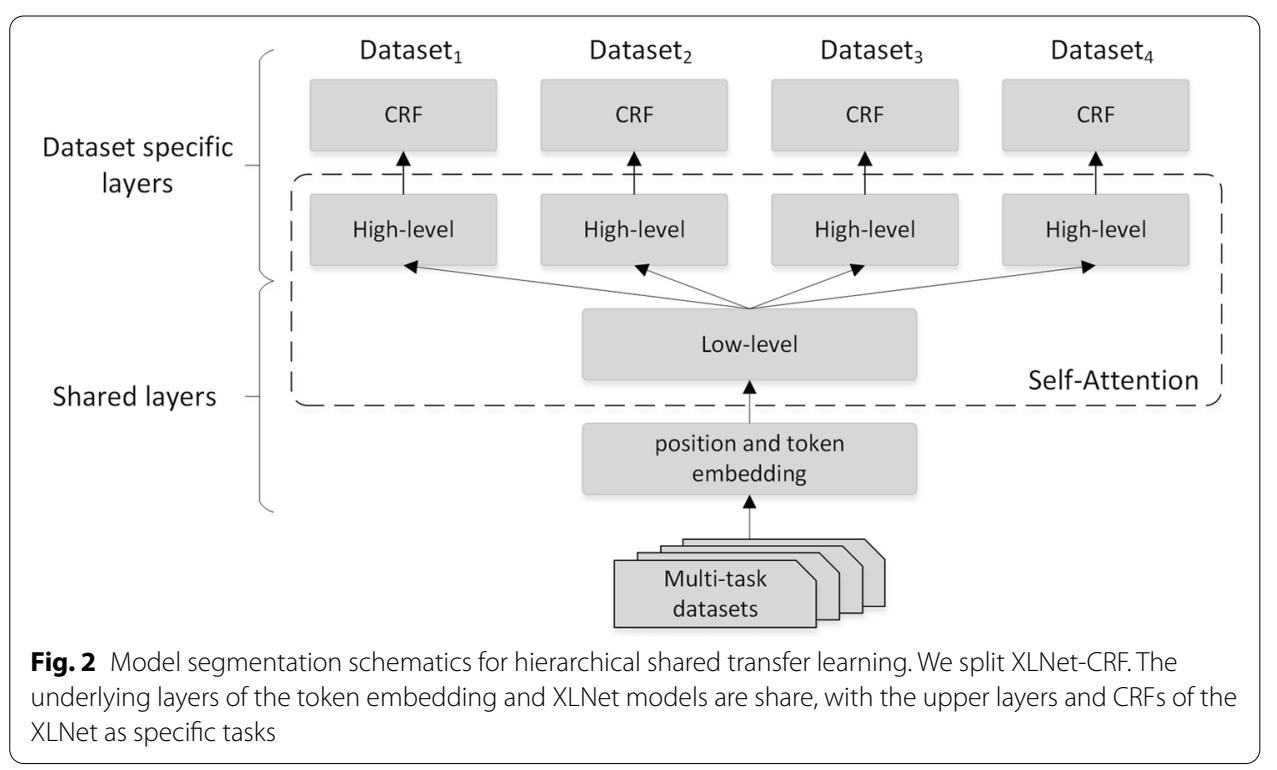


by layer. Fortunately, the parameters in the hidden layers of the XLNet model have the same output size, allowing them to be split and combined at will. Take $H^{k}$ as the dividing point between $H^{0}$ and $H^{n}$, define the underlying layer between $0 k$, and the layer between $k n$ is called the upper layer. Because the underlying contains the underlying text encoding information [31], we use the underlying as a shared layer, and the upper layer is a special task layer trained separately for different tasks. The underlying parameters are derived from the corresponding layer parameters of the MTL-XC training. The upper layer parameters are initialized by pre-trained parameters on the common corpus, which can accelerate the convergence of the model. CRF contains few parameters $\left(L^{2}\right)$ and is closest to the decoding layer, making it easier to train, so random initialization is still used so that the decoder can train the language characteristics of different tasks separately. It encodes and decodes specific tasks and retains the common encoding information learned by multi-task learning for a class of entities. Define the scale of the number of shared layers after split as the slicing rate (slicingrate $=k / n$ ), and when slicingrate $=0$, MTL-LS degrades to the singletask learning that is shared by the embedded table parameters. When slicingrate $=0$, this is similar to the method proposed Zuo and Zhang [21], where the model is divided into two parts: the encoder (XLNet) and the decoder (CRF). The encoder part is used as a shared layer, and the decoder part is used for specific datasets.

\section{Datasets and data preprocessing}

Using datasets similar to those in Crichton et al. [18]. We excluded AnatEM during the hierarchical shared transfer learning phase because the dataset was not in the 4 types of entities ultimately evaluated. Furthermore, we experimented on 14 other baseline datasets and divided the entities into four categories: gene/protein, chemical, disease, and species. We take BC5CDR [32], BC4CHEM [33], NCBI-disease [34], BC2GM [35] and LINNAEUS [36], five datasets are gold standard master datasets. We analyzed the relationship between training sets, test sets, and training effects for five gold standard datasets. These datasets are open and available from https://github.com/cambridgeltl/MTL-Bioinformatics-2016.

\section{Evaluation metrics}

Due to the limitation of the training cost, it is difficult to conduct multiple random initialization training, so instead, for each dataset, we first conducted $n$ epoch training sessions and then conducted $k * m$-round epoch training sessions with the obtained model parameters as the starting point. With the increment of $n, m$, the time cost becomes unacceptable and the convergence effect of the model has not been significantly improved. However, if $n, m$ is too small, also cannot converge. And finally we took a $30+3 * 30$ structure to train. In the test, the last five checkpoints of each training exercise were predicted against the test datasets. We calculated precision, recall, and F1-scores as evaluation indicators, with F1-scores as the primary evaluation indicators. The calculation formula is as follows:

$$
\begin{aligned}
& F 1-\text { score }=\frac{2 \cdot \text { precision } \cdot \text { recall }}{\text { precision }+ \text { recall }} \\
& \text { precision }=\frac{T P}{T P+F P}
\end{aligned}
$$




$$
\text { recall }=\frac{T P}{T P+F N}
$$

\section{Training detail}

XLNet-Large pre-training parameters provided by Yang et al. [6], which contain 24 layers, 1024 hidden layer nodes, and 16 attention heads. We're using Adam optimizer, Adam epsilon was $1 e-6$ and the learning rate was $3 e-5$, because the model was already pre-trained, so the model was not warmed-up during training. When multitasking, we unify the labels of the datasets and shuffle the datasets. In order to preserve the underlying shared information as much as possible, the layer attenuation strategy is used to reduce the learning rate of each layer, i.e. $\operatorname{lr}[n-1]=\operatorname{lr}[n] *$ decay_rate, where $n$ is the XLNet layer, and decay_rate $=0.9$.

\section{Results}

\section{Share all the parameters of the XLNet-CRF (MTL-XC)}

In this lab, the effects of four types of BioNER in MTL-XC were evaluated. Benchmark the results of training on a single-task. For datasets that have multiple entity types, we compare them separately into a single type. Table 1 provides a complete comparison of the performance of chemical, disease, species, and gene/protein on MTL-XC's precision, recall, F1-scores for 14 datasets. As can be seen from Table 1, the F1 of the two types of entities, disease, and gene/protein, has been greatly enhanced, and in the vast majority of the datasets, multi-task is better than single-task. Some datasets have been improved significantly, such as BioNLP13GE dataset by 5.37, Ex-PTM dataset by 6.73 and CRAFT dataset by 3.69. Although there has been an increase in disease entities, the increase has been relatively limited. In the remaining datasets, performance declines are severe. The same phenomenon occurs in the species category, where all datasets have a lower MTL$\mathrm{XC}$ results than single-task learning. Therefore, direct sharing of full model parameters is not ideal. Again, this proves that multi-tasking is not always better than single-task learning [28]. Entity categories and dataset features affect multi-tasking learning results, including association between dataset and size of datasets.

\section{Hierarchical shared transfer learning on XLNet-CRF (MTL-LS)}

We try to further improve the training effectiveness and stability of multi-task learning by layering the model. The new model is referred to as MTL-LS, as detailed in "Hierarchical shared transfer learning" section. Based on previous MTL-XC studies, we further trained fourteen datasets on MTL-LS. We made slicingrate $0.25,0.50,0.75$, and 1.00, respectively, as shown in Table 2.

We can see that the effects of different datasets are not stable with slicingrate. BC5CDR-chemical dataset results best when Slicingrate $=0.25$, but Slicingrate $=1.00$ in BC4CHEMD, which is also a chemical entity. BioNLP13PC-chemical and CRAFTchemical did not exceed single-task learning results but had a 5.93 and 5.91 improvement over multi-tasking, respectively. For disease entities, the BC5CDR-disease dataset has the largest F1 value at slicingrate $=1.00$, and the NCBI-disease dataset has the best effect at slicingrate $=0.75$. MTL-LS is better than single-tasks in all 10 
Table 1 Performance of STL-DS and MTL-XC on all tasks

\begin{tabular}{|c|c|c|c|c|c|c|}
\hline \multirow[t]{2}{*}{ Dataset } & \multicolumn{3}{|l|}{ STL } & \multicolumn{3}{|c|}{ MTL-XC } \\
\hline & P. $\%$ & R. $\%$ & F1 & P. \% & R. $\%$ & F1 \\
\hline \multicolumn{7}{|l|}{ Chemical } \\
\hline BC4CHEMD & 93.00 & 92.40 & 91.70 & 92.02 & 92.49 & 91.25 \\
\hline BC5CDR & 92.76 & 93.96 & 93.36 & 93.43 & 92.94 & 93.19 \\
\hline BioNLP11ID & 55.56 & 72.58 & 62.94 & 61.44 & 75.81 & 67.87 \\
\hline BioNLP13CG & 83.20 & 85.14 & 84.16 & 82.35 & 80.88 & 81.61 \\
\hline BioNLP13PC & 88.57 & 90.33 & 89.44 & 76.65 & 83.80 & 80.07 \\
\hline CRAFT & 84.07 & 81.05 & 82.54 & 75.34 & 72.38 & 73.83 \\
\hline \multicolumn{7}{|l|}{ Disease } \\
\hline $\mathrm{BC} 5 \mathrm{CDR}$ & 84.83 & 88.11 & 86.44 & 86.40 & 87.34 & 86.87 \\
\hline NCBI-disease & 87.27 & 89.27 & 88.26 & 87.80 & 89.73 & 88.75 \\
\hline \multicolumn{7}{|c|}{ Gene and protein } \\
\hline $\mathrm{BC} 2 \mathrm{GM}$ & 81.91 & 82.53 & 82.22 & 82.67 & 82.61 & 82.64 \\
\hline BioNLP09 & 88.20 & 86.82 & 87.50 & 87.23 & 91.95 & 89.53 \\
\hline BioNLP11EPI & 84.23 & 87.96 & 85.81 & 85.32 & 87.63 & 86.46 \\
\hline BioNLP11ID & 89.22 & 89.65 & 89.43 & 89.6 & 88.47 & 89.03 \\
\hline BioNLP13CG & 88.45 & 92.42 & 90.39 & 93.56 & 91.63 & 92.58 \\
\hline BioNLP13GE & 73.57 & 83.51 & 78.22 & 77.62 & 90.55 & 83.59 \\
\hline BioNLP13PC & 89.56 & 94.26 & 91.85 & 90.66 & 87.93 & 89.27 \\
\hline CRAFT & 80.48 & 75.44 & 77.88 & 78.56 & 84.83 & 81.57 \\
\hline Ex-PTM & 74.79 & 80.46 & 77.52 & 81.83 & 86.83 & 84.25 \\
\hline JNLPBA & 71.98 & 80.04 & 75.80 & 72.58 & 85.04 & 78.32 \\
\hline \multicolumn{7}{|l|}{ Species } \\
\hline BioNLP11ID & 85.41 & 82.03 & 83.68 & 91.22 & 70.24 & 79.37 \\
\hline BioNLP13CG & 88.34 & 89.19 & 88.76 & 88.39 & 86.68 & 87.52 \\
\hline CRAFT & 96.45 & 97.73 & 97.08 & 93.76 & 93.51 & 93.63 \\
\hline LINNAEUS & 91.70 & 85.62 & 88.56 & 88.43 & 82.14 & 85.17 \\
\hline
\end{tabular}

Better scores of each metric are in bold

sub-tasks of the gene/protein entity. In the species class, BioNLP11CG and CRAFT obtained the best results at the slicingrate of 0.25 and 1.00 respectively, while BioNLP13ID and LINNAEUS obtained the best results at the slicingrate of 0.75 . However, CRAFT-species and LINNAEUS dataset are slightly different, although the F1 value is higher than the MTL-XC, but they do not reach the F1 value achieved by single-task learning. In " $\operatorname{card}\left(C_{L} \cap P \cap T\right) / \operatorname{card}\left(C_{L} \cap P\right)$ and ( $\left.\operatorname{cardP}-\operatorname{cardL}\right) / \operatorname{cardL}$ " sections, we analyze the five gold standard dataset to find that the LINNAEUS data is of lower quality and smaller size, and that the introduction of other entity sets for training would reduce its $\mathrm{F} 1$ value.

The results show that MTL-LS is greatly affected by slicingrate and in some cases even learns less than STL. On the one hand, as slicingrate decreases, models converge more and more slowly. On the other hand, the relevance of the data itself makes it possible for the model to learn redundant noise information. But finding the right slicingrate makes the result except for JNLPBA better than all results on MTL-XC. Overall, training using MTL-LS resulted in a certain degree of steady improvement 
Table 2 F1 performance of STL, MTL-XC, and MTL-LS with different slicing rates

\begin{tabular}{|c|c|c|c|c|c|c|}
\hline \multirow[t]{2}{*}{ Dataset } & \multirow[t]{2}{*}{ STL } & \multirow[t]{2}{*}{ MTL-XC } & \multicolumn{4}{|c|}{ Slicng rate } \\
\hline & & & 0.25 & 0.50 & 0.75 & 1.00 \\
\hline \multicolumn{7}{|l|}{ Chemical } \\
\hline BC4CHEMD & 91.70 & 91.25 & 91.82 & 92.24 & 92.37 & 92.47 \\
\hline BC5CDR & 93.36 & 93.19 & 93.93 & 93.75 & 93.60 & 93.14 \\
\hline BioNLP11ID & 62.94 & 67.87 & 42.29 & 52.98 & 73.41 & 73.71 \\
\hline BioNLP13CG & 84.16 & 81.61 & 78.59 & 78.50 & 85.13 & 82.47 \\
\hline BioNLP13PC & 89.44 & 80.07 & 81.25 & 82.13 & 84.03 & 86.00 \\
\hline CRAFT & 82.54 & 73.83 & 76.73 & 79.74 & 77.96 & 79.44 \\
\hline \multicolumn{7}{|l|}{ Disease } \\
\hline BC5CDR & 86.44 & 86.87 & 86.10 & 87.04 & 86.28 & 87.34 \\
\hline NCBI-disease & 88.26 & 88.75 & 85.23 & 86.79 & 89.24 & 88.97 \\
\hline \multicolumn{7}{|c|}{ Gene and protein } \\
\hline $\mathrm{BC} 2 \mathrm{GM}$ & 82.22 & 82.64 & 79.85 & 81.54 & 81.94 & 82.94 \\
\hline BioNLP09 & 87.50 & 89.53 & 87.23 & 89.53 & 89.47 & 89.27 \\
\hline BioNLP11EPI & 85.81 & 86.46 & 84.65 & 85.65 & 86.24 & 86.58 \\
\hline BioNLP11ID & 89.43 & 89.03 & 83.62 & 82.69 & 89.59 & 86.55 \\
\hline BioNLP13CG & 90.39 & 92.58 & 91.33 & 92.05 & 92.71 & 92.21 \\
\hline BioNLP13GE & 78.22 & 83.59 & 81.29 & 82.61 & 82.73 & 84.03 \\
\hline BioNLP13PC & 91.85 & 89.27 & 89.16 & 91.87 & 90.59 & 90.37 \\
\hline CRAFT & 77.88 & 81.57 & 79.02 & 82.62 & 83.42 & 82.18 \\
\hline Ex-PTM & 77.52 & 84.25 & 78.52 & 80.57 & 84.64 & 84.55 \\
\hline JNLPBA & 75.80 & 78.32 & 75.08 & 77.00 & 77.32 & 77.66 \\
\hline \multicolumn{7}{|l|}{ Species } \\
\hline BioNLP11ID & 83.68 & 79.37 & 70.91 & 77.45 & 83.74 & 79.00 \\
\hline BioNLP13CG & 88.76 & 86.30 & 88.97 & 87.39 & 86.07 & 86.90 \\
\hline CRAFT & 97.08 & 93.63 & 95.56 & 95.04 & 94.74 & 95.75 \\
\hline LINNAEUS & 88.56 & 85.17 & 83.82 & 84.05 & 86.40 & 85.06 \\
\hline
\end{tabular}

Better scores of each metric are in bold

relative to MTL-XC. Sharing mechanisms at different levels can make it easier for a model to jump out of a local best.

Among the five sub-tasks for four gold standard datasets excluding LINNAEUS, the model presented in this paper has a significant advantage over the model based on LSTM [18, 37]. BC5CDR-chemical, BC5CDR-disease, BC2GM, BC4CHEMD, NCBIdisease compared to single-task XLNet models increased by 2.81, 1.67, 1.52, 1.18, 0.97 and 1.3 percentage points respectively. It can be concluded that the proposed MTL-LS architecture has better effect, generalization, and stability on BioNER.

\section{Comparison with benchmark results}

In this section, compare the results of MTL-LS for the five gold standard datasets with those of other the corresponding publications. The datasets we use are standard that already publicly available, so test splits are the same. To make a fair comparison with other people's work, we adjust the slicingrate on the development set of the data set and produce the final test set results, so that the slicingrate is not optimized on the test set. Take test set F1-score as shown in Table 3. 
Table 3 Model performance comparison to other studies

\begin{tabular}{|c|c|c|c|c|c|c|}
\hline & \multicolumn{2}{|l|}{ BC5CDR } & \multirow[t]{2}{*}{$\mathrm{BC} 2 \mathrm{GM}$} & \multirow[t]{2}{*}{ BC4CHEMD } & \multirow[t]{2}{*}{ NCBI-disease } & \multirow[t]{2}{*}{ LINNAEUS } \\
\hline & Chemical & Disease & & & & \\
\hline Crichton et al. [18] & 89.22 & 80.46 & 73.04 & 82.95 & 80.46 & 83.98 \\
\hline Yoon et al. [37] & 93.31 & 84.08 & 79.73 & 88.85 & 86.36 & - \\
\hline Lee et al. [26] & 93.44 & 86.56 & 84.40 & 91.41 & 89.36 & 89.81 \\
\hline BERT [26] & 91.16 & 82.41 & 81.79 & 90.04 & 85.63 & 87.60 \\
\hline PubWebBERT [27] & 93.33 & 85.62 & 84.52 & - & 87.82 & - \\
\hline HunFlair [38] & - & - & - & - & 88.65 & - \\
\hline STL & 93.36 & 86.44 & 82.52 & 91.70 & 88.26 & 88.56 \\
\hline Proposed & 93.83 & 87.28 & 82.92 & 92.42 & 89.25 & 86.37 \\
\hline
\end{tabular}

Better scores of each metric are in bold

In general, the methods presented in this paper perform poorly on the LINNAEUS dataset. After analysis, the model can easily converge with the local optimal when training LINNAEUS and has not found a way to improve the effect. Except LINNAEUS, single-task training results were better than the BERT. The XLNet-CRF single-task is not good enough compared to PubWebBERT with only BC2GM. NCBI-disease is better at STL without HunFlair, but MTL-LS is better than HunFlair. BioBERT achieved more than single-task training through further pre-training in medical data and exceeded the model proposed in this paper on BC2GM, NCBI-release and LINNAEUS. However, hierarchical shared transfer learning outperforms BioBERT models on BC5CDR and BC4CHEMD datasets. It can be argued that pre-training of knowledge data in biomedical fields can significantly improve entity identification of genes/proteins and species classifications. For other entity types, it is better to combine multitasking with fine-tuning.

\section{Discussion}

Multi-task learning essentially increases the generalization of the model by increasing the number of training samples to cover as many entities as possible. Therefore, the correlation of data in multi-task learning often greatly affects the effect of training. If the data is less dependent, the F1 value is lowered. We counted the entities of five dataset, where the multi-task learning entity set Training is represented by $T$, and the test set contains the entity set Labels represented by $L$ and the entity set Logits predicted by the final model are represented by $P$, shown in Fig. 3 as a Euler graph. We compute the number of parts of the set, shown in Fig. 4 as a radar chart.

$\operatorname{card}\left(C_{T} \cap L \cap P\right) / \operatorname{card}\left(C_{T} \cap L\right)$

We take BC4CHEMD as an example to describe the meaning of the Euler diagram, and $C_{T} \cap L(2.30 \%+18.37 \%)$ indicates a set of entities that need to be predicted but not trained. $C_{T} \cap L \cap P(18.37 \%)$ denotes the set of entities that are not in the training set, but that predict success. The greater the $\operatorname{card}\left(C_{T} \cap L \cap P\right) / \operatorname{card}\left(C_{T} \cap L\right)$, the more adaptable the model is. We present the $\operatorname{card}\left(C_{T} \cap L \cap P\right) / \operatorname{card}\left(C_{T} \cap L\right)$ of the six datasets as a radar chart in Fig. 4A. The figure shows that this percentage of BC4CHEMD reached $88.86 \%$, which explains why the F1 value of BC4CHEMD can reach $92.47 \%$. The 

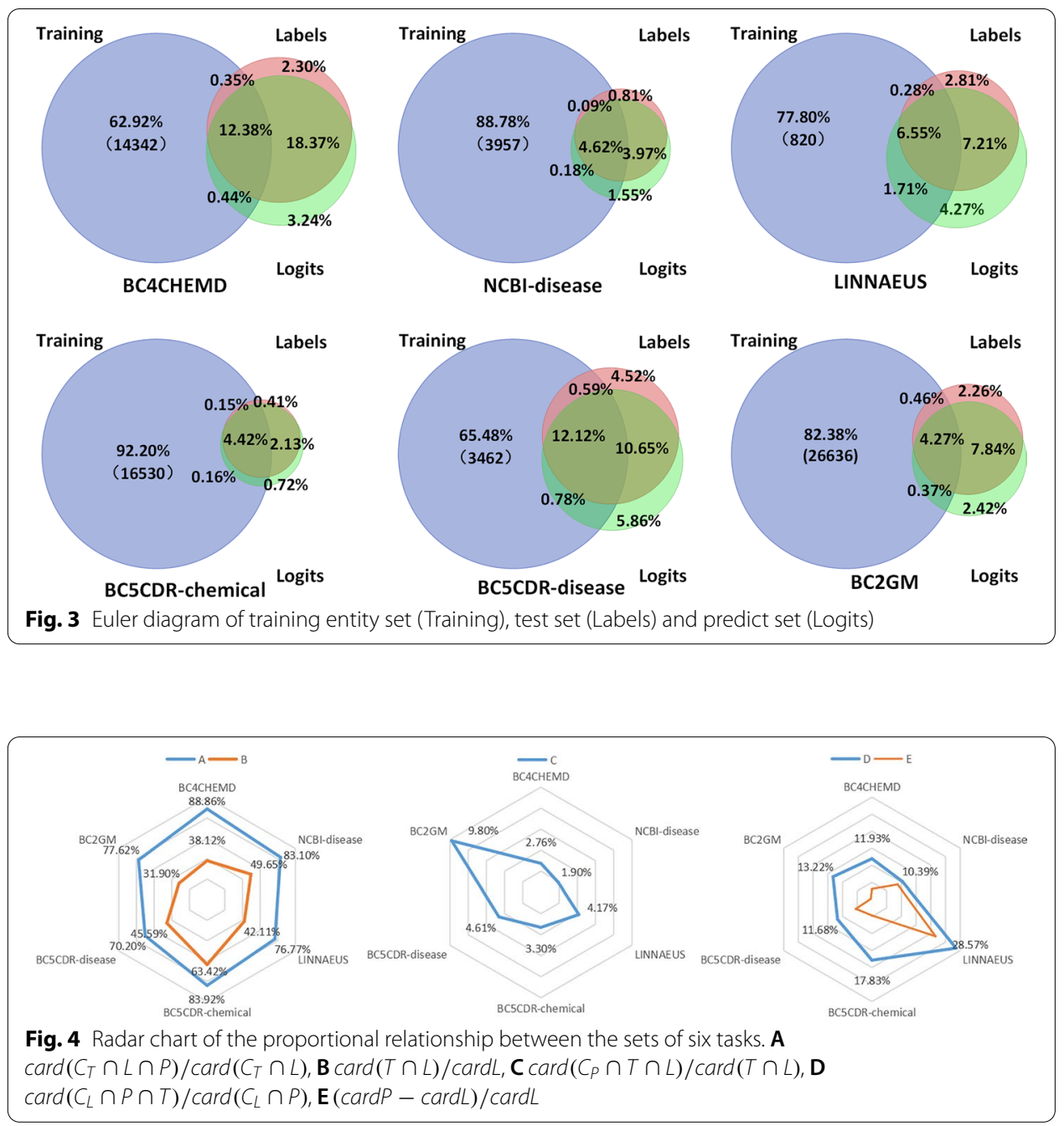

average value of $\operatorname{card}\left(C_{T} \cap L \cap P\right) / \operatorname{card}\left(C_{T} \cap L\right)$ can reach $80.08 \%$, which is a good indication that our model learns a certain amount of knowledge, has some learning ability, and can transfer learning.

$\operatorname{card}(T \cap L) / \operatorname{cardL}$

Figure 4B indicates that $\operatorname{card}(T \cap L) / \operatorname{cardL}, T \cap L$ represents the intersection of the training set and the test set entities. The greater this value, the greater the proportion of entities covered by the training set, the greater the generalization of the model. The average can reach $45.13 \%$, BC5CDR-chemical $\operatorname{card}(T \cap L) / \operatorname{cardL}$ even reached $63.42 \%$.

$\operatorname{card}\left(C_{P} \cap T \cap L\right) / \operatorname{card}(T \cap L)$

Figure $4 \mathrm{C}$ shows $\operatorname{card}\left(C_{P} \cap T \cap L\right) / \operatorname{card}(T \cap L)$, where $C_{P} \cap T \cap L$ is the set of entities that have been learned by the training set but cannot be identified in the test set, which represents an average of $4.42 \%$ of $T \cap L$. This phenomenon suggests that even learned knowledge can be forgotten, and the introduction of a larger 
number of samples can distract the model, resulting in a loss of memory and a lack of recognition of the learned entity. We can see from the radar chart that the value of card $\operatorname{card}\left(C_{P} \cap T \cap L\right) / \operatorname{card}(T \cap L)$ for BC2GM reached 9.8\%. Second, BC2GM mixes ten task genes/protein entities of $\operatorname{card}(T \cap L) / \operatorname{cardL}$, (Fig. 4B) and only $31.90 \%$. For BC2GM, which forgets $9.8 \%$ of the knowledge and has low coverage, the F1 value is the worst in the six datasets.

$\operatorname{card}\left(C_{L} \cap P \cap T\right) / \operatorname{card}\left(C_{L} \cap P\right)$

Another particular note is the radar chart shown in $\operatorname{card}\left(C_{L} \cap P \cap T\right) / \operatorname{card}\left(C_{L} \cap P\right)$ in Fig. 4D. $C_{L} \cap P$ represents the set of entities that recognize the error, and $C_{L} \cap P \cap T$ and $C_{L} \cap P \cap C_{T}$ are included in $C_{L} \cap P \cap T$, where $C_{L} \cap P \cap T$ is very noteworthy. The entity set is treated as the entity training in the training set, but in the test set $C_{L} \cap P \cap T$ is not the entity, that is, the wrong knowledge is learned when learning, or the model identifies the accurate entity according to the prior knowledge, and the test set tells the model that the prediction error is contradictory. Therefore, the larger $C_{L} \cap P \cap T$ as $C_{L} \cap P$, the worse the quality of the dataset. Figure 4D shows that this problem exists in all six tasks, with an average of $15.6 \%$. That is, the dataset itself has some errors. And the LINNAEUS dataset has $28.57 \%$ error recognition from training data, which is why LINNAEUS's single-task results are much better than multi-task results. The introduction of new training samples increases the probability of model error. The relevance of datasets in species categories can be considered to have contributed to this result. There is a need to improve the quality of data for this type of dataset to avoid problems with misperception.

(cardP - cardL)/cardL

As shown in Fig. $4 \mathrm{E}$, the ( $\operatorname{cardP}-\operatorname{cardL}) / \operatorname{cardL}$ values are greater than 0 , that is, the number of entities predicted by all sets is greater than the original number of entities in the test set. The LINNAEUS dataset has a value of $21.64 \%$, which again explains why the LINNAEUS multi-task F1 value never reaches the effect of a single-task. For LINNAEUS, our model has learned a lot that is not its own.

In conclusion, we analyze the relationship between training sets, test sets, and entity sets of predicted results, and point out the error sources at the data level. This paper explains the reason the experiment results of LINNAEUS dataset can't transcend the single-task at the data level.

\section{Conclusion}

Because of the previous methods of biomedical named entity recognition through deep learning methods, single-task learning or multi-task learning. This paper presents an effective hierarchical shared transfer learning method, which combines multi-task with single-task, has high generalization and stability, and validates its effectiveness on fourteen datasets. In addition, we analyzed the physical relationship between training sets, test sets, and training effects on five gold standard datasets. The source of error at the data level is pointed out. 


\section{Abbreviations}

BioNER: Biomedical named entity recognition; CRF: Conditional random field; CNN: Convolutional neural networks; BiLSTM: Bidirectional long short-term memory; BERT: Bidirectional encoder representations from transformers; AutoEncoder LM: AutoEncoding language model; OOV: Out-of-vocabulary; MTL: Multi-task learning; STL: Single-task learning; MTL-XC: Share all the parameters of the XLNet-CRF; MTL-LS: Hierarchical shared transfer learning on XLNet-CRF.

\section{Acknowledgements}

Not applicable.

\section{Authors' contributions}

$\mathrm{ZC}$ and $\mathrm{HJ}$ designed and implemented the overall framework and conducted the experiments, and was a major contributor in writing the manuscript. SHS provided funding acquisition, project administration, manuscript revisions and conceptual oversight. SYZ provided funding acquisition, project administration, writing and revising the manuscript; $L Z$ and $Y Y$ were major contributors in writing and revising the manuscript. All authors read and approved the final manuscript.

\section{Funding}

This work has been supported by the Ministry of Science and Technology of the People's Republic of China (No. 2021YFC0863400), and the National Natural Science Foundation of China (No. 81973146).

Availability of data and materials

XLNet-Large pre-training parameters were provided by [https://storage.googleapis.com/xlnet/released_models/ cased_L-24_H-1024_A-16.zip]

\section{Declarations}

Ethics approval and consent to participate

Not applicable.

\section{Consent for publication}

Not applicable.

\section{Competing interests}

The authors declare that they have no competing interests.

\section{Author details}

${ }^{1}$ College of Information Science and Technology, Beijing University of Chemical Technology, Beijing, China. ${ }^{2}$ School of Public Health, Peking University, Beijing, China. ${ }^{3}$ Research Center of Clinical Epidemiology, Peking University Third Hospital, Beijing, China. ${ }^{4}$ National Institute of Health Data Science, Peking University, Beijing, China.

Received: 14 October 2021 Accepted: 22 December 2021

Published online: 04 January 2022

\section{References}

1. Savova GK, Masanz JJ, Ogren PV, Zheng J, Sohn S, Kipper-Schuler KC, Chute CG. Mayo clinical Text Analysis and Knowledge Extraction System (CTAKES): architecture, component evaluation and applications. J Am Med Inform Assoc. 2010;17(5):507-13. https://doi.org/10.1136/jamia.2009.001560.

2. Shickel B, Tighe PJ, Bihorac A, Rashidi P. Deep EHR: a survey of recent advances in deep learning techniques for electronic health record (EHR) analysis. IEEE J Biomed Health Inform. 2018;22(5):1589-604. https://doi.org/10.1109/ JBHI.2017.2767063. arXiv:1706.03446.

3. LeCun Y, Boser B, Denker JS, Henderson D, Howard RE, Hubbard W, Jackel LD. Backpropagation applied to handwritten zip code recognition. Neural Comput. 1989;1 (4):541-51. https://doi.org/10.1162/neco.1989.1.4.541.

4. Malhotra P, Vig L, Shroff G, Agarwal P. Long short term memory networks for anomaly detection in time series. In: 23rd European symposium on artificial neural networks, computational intelligence and machine learning, ESANN 2015-proceedings (April), 2015. p. 89-94

5. Mehmood T, Gerevini AE, Lavelli A, Serina I. Combining multi-task learning with transfer learning for biomedical named entity recognition. Procedia Comput Sci. 2020;176:848-57. https://doi.org/10.1016/j.procs.2020.09.080.

6. Yang Z, Dai Z, Yang Y, Carbonell J, Salakhutdinov R, Le QV. XLNet: Generalized autoregressive pretraining for language understanding. Adv Neural Inf Process Syst. 2019;32(NeurlPS):1-18 arXiv:1906.08237.

7. Zhuang F, Qi Z, Duan K, Xi D, Zhu Y, Zhu H, Xiong H, He Q. A Comprehensive Survey on Transfer Learning. Proceedings of the IEEE. 2021:109(1):43-76. https://doi.org/10.1109/JPROC.2020.3004555. arXiv:1911.02685.

8. Wang YS, Zhang ZF, Li MH, Zhang P, Liu XY. Universal language model fine-tuning for text classification. Chin J Ophthalmol. 2010;46(2):119-24. https://doi.org/10.3760/cma.j.issn.04124081.2010.02.006.

9. Tan C, Sun F, Kong T, Zhang W, Yang C, Liu C. A survey on deep transfer learning. In: Lecture notes in computer science (including subseries lecture notes in artificial intelligence and lecture notes in bioinformatics) 11141 LNCS, 2018. p. 270-279. https://doi.org/10.1007/978-3-030-01424-7_27. arXiv:1808.01974

10. Fausk $\mathrm{H}$, Isaksen DC. Improving language understanding by generative pre-training. Homol Homot Appl. 2007:9(1):399-438. https://doi.org/10.4310/HHA.2007.v9.n1.a16.

11. Giorgi JM, Bader GD. Transfer learning for biomedical named entity recognition with neural networks. Bioinformatics. 2018;34(23):4087-94. https://doi.org/10.1093/bioinformatics/bty449. 
12. Wu C, Xiao X, Yang C, Chen JX, Yi J, Qiu Y. Mining microbe-disease interactions from literature via a transfer learning model. BMC Bioinform. 2021;22(1):1-15. https://doi.org/10.1186/s12859-021-04346-7.

13. Wang Y, Xia Z, Deng J, Xie X, Gong M, Ma X. TLGP: a flexible transfer learning algorithm for gene prioritization based on heterogeneous source domain. BMC Bioinform. 2021;22(9):1-16. https://doi.org/10.1186/s12859-021-04190-9.

14. CY, Genc S, Chung J, Sun T, Mallya S. REPAINT: Knowledge transfer in deep reinforcement learning 2020. arXiv:2011. 11827

15. Joshi A, Karimi S, Sparks R, Paris C, MacIntyre CR. Does multi-task learning always help? an evaluation on health informatics. In: Proceedings of the the 17 th annual workshop of the Australasian language technology association, 2019. p. 151-158

16. Li J, Sun A, Han J, Li C. A survey on deep learning for named entity recognition. IEEE Trans Knowl Data Eng. 2020:XX(XX):1. https://doi.org/10.1109/tkde.2020.2981314. arXiv:1812.09449.

17. Naik A, Rangwala H. Multi-task learning. SpringerBriefs Comput Sci. 2018;75:75-88. https://doi.org/10.1007/ 978-3-030-01620-3_5.

18. Crichton G, Pyysalo S, Chiu B, Korhonen A. A neural network multi-task learning approach to biomedical named entity recognition. BMC Bioinform. 2017;18(1):1-14. https://doi.org/10.1186/s12859-017-1776-8.

19. Habibi M, Weber L, Neves M, Wiegandt DL, Leser U. Deep learning with word embeddings improves biomedical named entity recognition. Bioinformatics. 2017;33(14):37-48. https://doi.org/10.1093/bioinformatics/btx228.

20. Wang $X$, Zhang Y, Ren X, Zhang Y, Zitnik M, Shang J, Langlotz C, Han J. Cross-type biomedical named entity recognition with deep multi-task learning. Bioinformatics. 2019;35(10):1745-52. https://doi.org/10.1093/bioinformatics/ bty869. arXiv:1801.09851.

21. Zuo M, Zhang Y. Dataset-aware multi-task learning approaches for biomedical named entity recognition. Bioinformatics. 2020;36(15):4331-8. https://doi.org/10.1093/bioinformatics/btaa515.

22. Dai AM, Le QV. Semi-supervised sequence learning. Adv Neural Inf Process Syst. 2015;2015:3079-87 arXiv:1511.01432.

23. No S. Deep contextualized word representations. Naacl 2018;2227-2237. arXiv:1802.05365

24. Vaswani A, Shazeer N, Parmar N, Uszkoreit J, Jones L, Gomez AN, Kaiser Ł, Polosukhin I. Attention is all you need. Adv Neural Inf Process Syst. 2017;2017:5999-6009 arXiv:1706.03762.

25. Devlin J, Chang MW, Lee K, Toutanova K. BERT: Pre-training of deep bidirectional transformers for language understanding. In: NAACL HLT 2019-2019 conference of the North American chapter of the association for computational linguistics: human language technologies_-proceedings of the conference 1(MIm), 2019. p. 4171-4186. arXiv:1810. 04805

26. Lee J, Yoon W, Kim S, Kim D, Kim S, So CH, Kang J. BioBERT: A pre-trained biomedical language representation model for biomedical text mining. Bioinformatics. 2020;36(4):1234-40. https://doi.org/10.1093/bioinformatics/btz682. arXiv:1901.08746.

27. GuY, Tinn R, Cheng H, Lucas M, Usuyama N, Liu X, Naumann T, Gao J, Poon H. Domain-specific language model pretraining for biomedical natural language processing. ACM Trans Comput Healthc. 2022;3(1):1-23. https://doi.org/ 10.1145/3458754. arXiv:2007.15779.

28. Yao L, Jin Z, Mao C, Zhang Y, Luo Y. Traditional Chinese medicine clinical records classification with BERT and domain specific corpora. J Am Med Inform Assoc. 2019;26(12):1632-6. https://doi.org/10.1093/jamia/ocz164.

29. Pulford G. The viterbi algorithm. IET Seminar Dig. 2006;2006(11359):53-65. https://doi.org/10.1049/ic:20060556.

30. Giorgi JM, Bader GD. Towards reliable named entity recognition in the biomedical domain. Bioinformatics. 2020;36(1):280-6. https://doi.org/10.1093/bioinformatics/btz504.

31. Søgaard A, Goldberg Y. Deep multi-task learning with low level tasks supervised at lower layers. In: 54 th Annual meeting of the association for computational linguistics, ACL 2016-short papers, 2016. p. 231-235. https://doi.org/ $10.18653 / \mathrm{v} 1 / \mathrm{p} 16-2038$

32. Li J, Sun Y, Johnson RJ, Sciaky D, Wei CH, Leaman R, Davis AP, Mattingly CJ, Wiegers TC, Lu Z. BioCreative V CDR task corpus: a resource for chemical disease relation extraction. Database J Biol Databases Curation. 2016;2016:1-10. https://doi.org/10.1093/database/baw068.

33. Krallinger M, Rabal O, Leitner F, Vazquez M, Salgado D, Lu Z, Leaman R, Lu Y, Ji D, Lowe DM, Sayle RA, Batista-Navarro RT, Rak R, Huber T, Rocktäschel T, Matos S, Campos D, Tang B, Xu H, Munkhdalai T, Ryu KH, Ramanan SV, Nathan S, Žitnik S, Bajec M, Weber L, Irmer M, Akhondi SA, Kors JA, Xu S, An X, Sikdar UK, Ekbal A, Yoshioka M, Dieb TM, Choi M, Verspoor K, Khabsa M, Giles CL, Liu H, Ravikumar KE, Lamurias A, Couto FM, Dai HJ, Tsai RTH, Ata C, Can T, Usié A, Alves R, Segura-Bedmar I, Martínez P, Oyarzabal J, Valencia A. The CHEMDNER corpus of chemicals and drugs and its annotation principles. J Cheminform. 2015;7(Suppl 1):1-17. https://doi.org/10.1186/1758-2946-7-S1-S2.

34. Doğan RI, Leaman R, Lu Z. NCBI disease corpus: a resource for disease name recognition and concept normalization. J Biomed Inform. 2014;47:1-10. https://doi.org/10.1016/j.jbi.2013.12.006

35. Smith L, Tanabe LK, Ando R, Kuo CJ, Chung IF, Hsu CN, Lin YS, Klinger R, Friedrich CM, Ganchev K, Torii M, Liu H, Haddow B, Struble CA, Povinelli RJ, Vlachos A, Baumgartner WA, Hunter L, Carpenter B, Tsai RTH, Dai HJ, Liu F, Chen Y, Sun C, Katrenko S, Adriaans P, Blaschke C, Torres R, Neves M, Nakov P, Divoli A, Maña-López M, Mata J, Willbur WJ. Overview of BioCreative II gene mention recognition. Genome Biol. 2008;9(SUPPL. 2):1-19. https://doi.org/10.1186/ gb-2008-9-s2-s2.

36. Gerner M, Nenadic G, Bergman CM. LINNAEUS: A species name identification system for biomedical literature. BMC Bioinform. 2010. https://doi.org/10.1186/1471-2105-11-85.

37. Yoon W, So CH, Lee J, Kang J. CollaboNet: Collaboration of deep neural networks for biomedical named entity recognition. BMC Bioinform. 2019. https://doi.org/10.1186/s12859-019-2813-6. arXiv:1809.07950.

38. Weber L, Sänger M, Münchmeyer J, Habibi M, Leser U, Akbik A. HunFlair: An easy-to-use tool for state-of-the-art biomedical named entity recognition. Bioinformatics. 2021;37(17):2792-4. https://doi.org/10.1093/bioinformatics/ btab042. arXiv:2008.07347.

\section{Publisher's Note}

Springer Nature remains neutral with regard to jurisdictional claims in published maps and institutional affiliations. 